\title{
Mott and pseudogap localization in pressurized $\mathrm{NbO}_{2}$
}

\author{
L. Craco ${ }^{1}$ and S. Leoni ${ }^{2}$ \\ ${ }^{1}$ Instituto de Física, Universidade Federal de Mato Grosso, 78060-900, Cuiabá, Mato Grosso, Brazil \\ ${ }^{2}$ School of Chemistry, Cardiff University, Cardiff, CF10 3AT, United Kingdom
}

(Received 20 May 2020; revised 1 July 2020; accepted 14 July 2020; published 24 July 2020)

\begin{abstract}
We present a detailed study of correlation-induced electronic reconstruction in baddeleyite-type $\mathrm{NbO}_{2}$, a distorted $\mathrm{ZrO}_{2}$-type structure that is found at pressures above $8.0 \mathrm{GPa}$. Based on density-functional plus dynamical mean-field theory (DFT+DMFT), we stress the importance of multiorbital Coulomb interactions in concert with first-principles band-structure calculations for a consistent understanding of emergent Mottness and pseudogap behavior in pressurized $\mathrm{NbO}_{2}$ and related $d^{1}$ systems. After a proper treatment of multiorbital electron-electron interactions, we find a nearly universal Mott behavior for the peak position of the lower Hubbard band that is independent of crystal- and band-structure details. We explain the nature of the metalpseudogap-insulator transition to be seen in experiment, showing a first-order transition between two metallic states: a correlated metal and a pseudogap state with a deep density of states at the Fermi level. This emergent pseudogap phenomena is expected to play a central role toward quantum criticality in pressurized $\mathrm{NbO}_{2}$.
\end{abstract}

DOI: 10.1103/PhysRevB.102.045142

\section{INTRODUCTION}

Electron correlation-driven metal-insulator transitions in transition-metal oxides remains a problem of enduring interest in condensed-matter physics [1]. Vanadium oxides have proven to be fascinating candidates in this context [2]. A particularly interesting member of this class is vanadium dioxide $\left(\mathrm{VO}_{2}\right)$, which undergoes a first-order metal-insulator transition on lowering temperature [3]. The high- $T$ metallic phase has a tetragonal rutile-type structure, which is reduced to monoclinic due to a first-order transition occurring around 340 K. Mott (electron correlation) [4] and Peierls (structural distortion) [5] physics are understood to play essential roles, and possibly to interplay in this transition. Accordingly, the observed first-order transition in $\mathrm{VO}_{2}$ is now dubbed MottPeierls, meaning that the lattice distortion is assisted [6] by the presence of strongly correlated electronic states in close proximity to Mottness [7,8]. While this interplay may be suppressed in $\mathrm{VO}_{2}$ ultrathin films [9], dynamical scatterings arising from multiorbital (MO) electron-electron interactions should be taken into consideration as an additional ingredient toward the formation of exotic phases in response to external perturbations and growth conditions. In this paper, along this line, we show that a dynamically reconstructed many-particle state induced by MO many-particle interactions [10] also holds true for the high-pressure structural phase of $\mathrm{NbO}_{2}$ [11]. As in other real $d$-band materials, both local geometric distortions and intrinsic electron-electron interactions can significantly affect their electronic-structure reconstruction, in particular, the occupation and energy position of the $d$-shell levels. Within the local-density-approximation plus dynamical-mean-field-theory (LDA+DMFT) framework [12], in this paper we show how the active $4 d$ orbitals of pressurized $\mathrm{NbO}_{2}$ are reconstructed relative to their bare band values. To advance our understanding of strongly correlated Mott-Peierls systems [6-8], we focus on the baddeleyite-type structural phase of $\mathrm{NbO}_{2}$ [11]. In spite of earlier studies $[13,14]$, the question we tackle here is how the electronic structure of $\mathrm{NbO}_{2}$ [15] is dynamically reshaped by the interplay between $\mathrm{MO}$ dynamic electronic correlations and sizable lattice distortions under pressure, showing an emergent pseudogap regime as the precursor of the Mott localized state [16] in undoped $\mathrm{NbO}_{2}$ at high pressure conditions. Along this line, we uncover a universal behavior of electronic reconstruction, extending beyond the compound of interest.

Under ambient pressure conditions, $\mathrm{NbO}_{2}$ undergoes a metal-insulator transition at $1081 \mathrm{~K}$, accompanied by a structural transition from rutile to a distorted body-centered tetragonal (bct) lattice [17]. Despite the differences in long-range order, local deviations from rutile are similar as found in $\mathrm{VO}_{2}$, since across the transition the dimerized $\mathrm{Nb}$ atoms also experience lateral displacements [18]. Interestingly, an additional transition has been observed above $8.0 \mathrm{GPa}$ to a monoclinic (baddeleyite or $\mathrm{ZrO}_{2}$-related) structure (space group $P 2_{1} / c$ ) with cell constants (at 8.3 GPa): $a=10.029(2) \AA$, $b=10.00(1) \AA, c=9.987(1) \AA$, and $\beta=104.023(4)^{\circ}$ [11]. Upon decompression, at $5 \mathrm{GPa}, \beta$ has narrowed to $\beta=$ $103.1^{\circ}$, under elongation of the $c$ axis. This indicates that the monoclinic angle is a sensible parameter, one that allows the progress of the structural transition to be monitored. The pressurized crystal structure with the doubling of the unit cell along $a, b, c$, and the corresponding monoclinic angle $(\beta)$ is shown in Fig. 1. Importantly, resistance data of $\mathrm{NbO}_{2}$ under pressure shows a sharp drop close to $85 \mathrm{kbar}$ accompanied by a first-order reduction of its activation energy [19]. With this in place, in this paper, we provide a correlated many-particle description of intrinsic Mott localization in baddeleyite-type $\mathrm{NbO}_{2}$ (here dubbed $\gamma-\mathrm{NbO}_{2}$ ), showing how its electronic state is reshaped upon small changes of $\beta$ under pressure.

In $\mathrm{NbO}_{2}$, the role of electron-electron interactions on the electronic properties of the rutile and bct phases were 


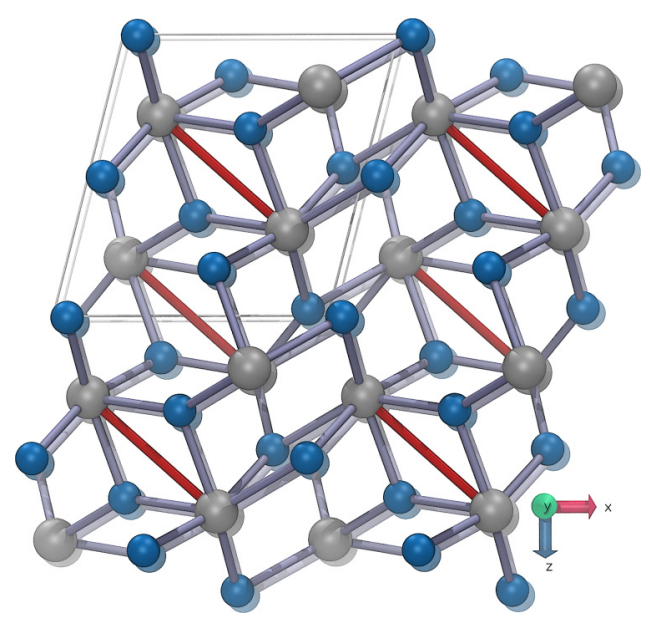

FIG. 1. Crystal structure of baddeleyite-type $\mathrm{NbO}_{2}$ after optimization for a choice of the monoclinic angle $\beta=104.29^{\circ} . \mathrm{Nb}$ and $\mathrm{O}$ are represented as large (silver) and small (blue) spheres, respectively. To illustrate the relative arrangement of atoms in the distorted octahedral coordination of pressurized $\mathrm{NbO}_{2}$, the origin of the unit cell is chosen at $\mathrm{O}$ positions. Short $\mathrm{Nb}-\mathrm{Nb}$ contacts are shown as dark-red sticks.

addressed in Ref. [13]. According to this cluster density functional dynamical mean-field study (cluster DFT+DMFT) within the rutile-type phase, electronic correlations lead to strong renormalization of the $t_{2 g}$ bands and the emergence of incoherent Hubbard bands at high energies. That study also finds the presence of sizable intersite electronic correlations within the dimerized bct phase. According to their description, these nonlocal correlations are important in the band-gap formation of insulating phases, with similarities akin to $\mathrm{VO}_{2}$ [8]. Consistent with an early study [20], their results suggest that structural distortions are not solely responsible for the gap opening of the bct phase, ruling out a purely Peierlstype nature for this insulating phase at ambient pressure. Moreover, the role played by $\mathrm{MO}$ electronic correlations within the rutile phase of normal and strained $\mathrm{NbO}_{2}$ were also more recently reported [14], showing that while the band structure is modified by the elongation of the $c$ axis driving spectral weight transfer into the $d_{x^{2}-y^{2}}$ orbital due to interband and interorbital hybridization, electron correlations were found to distribute electrons more evenly among the three $t_{2 g}$ orbitals. According to this DFT plus slave-spin study, the quasiparticle weights of the active orbitals are nearly the same in normal and strained $\mathrm{NbO}_{2}$, which is different from what they have found for $\mathrm{VO}_{2}$. This result was attributed to the fact that the bandwidth of the $d$ orbitals in $\mathrm{NbO}_{2}$ is larger, compared to $\mathrm{VO}_{2}$, implying smaller interaction to bandwidth ratio $(U / W)$ in the $4 d$ electronic state of $\mathrm{NbO}_{2}$. So far, however, the role played by MO electron-electron interactions in the electronic-structure reconstruction and gap formation within the high-pressure baddeleyite phase has not been addressed. In this paper, we shed light onto this problem, showing that $\mathrm{NbO}_{2}$ is an ideal $4 d^{1}$ material to study electronic localization under high-pressure conditions [19].

\section{RESULTS AND DISCUSSION}

$\mathrm{NbO}_{2}$ shares structural similarities with $\mathrm{TiO}_{2}$ and $\mathrm{ZrO}_{2}$ in the lower pressure regime. For both the rutile-type and the baddeleyite-type phases, the monoclinic angle (space group $P 2_{1} / c$ ) is always lower in $\mathrm{TiO}_{2}$ than in $\mathrm{NbO}_{2}$. This is attributed [11] to a stronger tendency to form short metal-metal contacts in the latter, which affects the monoclinic angle and in turn the $c$ axis module. Therefore, we have studied the structural evolution of $\gamma-\mathrm{NbO}_{2}$ as a function of the monoclinic angle, for a pressure above $8 \mathrm{GPa}(8.8 \mathrm{GPa})$, consistent with experiments [11]. Isotype $\mathrm{ZrO}_{2}$ provided the initial position coordinates and the cell vector moduli [21]. The monoclinic angles were chosen at $\beta=99.2^{\circ}$ (baddeleyitetype $\mathrm{TiO}_{2}$ ) and $\beta=104.2^{\circ}$ (baddeleyite-type $\mathrm{NbO}_{2}$ ), with an intermediate value of $\beta=101.75^{\circ}$. For each geometry optimization, only the $\beta$ angle was initially kept unchanged to allow positions and cell modules to partially relax (atomic forces $<0.02 \mathrm{eV} / \AA$ ) , followed by full parameter optimization (forces $<0.005 \mathrm{eV} / \AA$ ). The obtained final angles were $\beta=$ $99.28^{\circ}, 101.78^{\circ}$, and $104.29^{\circ}$, respectively. All three structures maintained the initial space group symmetry after geometry optimization. The volume contracted as a function of the angle, i.e., larger angle values favor smaller volumes: $V=$ $126.16 \AA^{3}$ for $\beta=104.29^{\circ}$ vs $126.42 \AA^{3}$ for $\beta=99.28^{\circ}$. On lowering pressure (not shown), the $c$-axis module increases and the monoclinic angle is reduced.

Within the monoclinic $\left(\mathrm{ZrO}_{2}\right.$-type) structure [11], see Fig. 1, one-electron band-structure calculations based on LDA were performed for the high-pressure phase of $\mathrm{NbO}_{2}$ using the linear muffin-tin orbitals scheme [22] in the atomic sphere approximation: Self-consistency is reached by performing calculations with 3770 irreducible $\mathbf{k}$ points. The radii of the atomic spheres were chosen as $r=2.67(\mathrm{Nb}), r=1.94(\mathrm{O} 1)$, and $r=2.16(\mathrm{O} 2)$ a.u. to minimize their overlap. Consistent with previous calculations for $\mathrm{NbO}_{2}$ [13-15], our LDA results in Fig. 2 confirms that the active electronic states in $\gamma-\mathrm{NbO}_{2}$ involve the $\mathrm{Nb} 4 d$ carriers. Similar to Ref.[14], here we follow the local coordinate system introduced in Ref. [15], in which the $t_{2 g}$ orbitals are $d_{x^{2}-y^{2}}\left(d_{\|}\right), d_{x z}$, and $d_{y z}\left(d_{\pi}\right.$ bands), and the $e_{g}$ orbitals are labeled as $d_{3 z^{2}-r^{2}}$ and $d_{x y}$. Interestingly, our results are consistent with extant calculations for rutile $\mathrm{NbO}_{2}$ [15], showing that lowest $t_{2 g}$ bands have predominant spectral weight close to $E_{F}=\omega=0$. While in metallic rutile the $d_{x z}$ orbital also partially contributes at $E_{F}$, in $\gamma-\mathrm{NbO}_{2}$ this electronic channel shifts to higher energies due to crystal field splitting inducing strong hybridization with the high in energy $e_{g}$ orbitals. Thus, as compared to the rutile phase, changes in crystal field splitting under pressure causes nearly complete energy separation of the $\mathrm{Nb} 4 d$ bands into two groups: a low in energy $x^{2}-y^{2}, y z$ group contributing near $E_{F}$ and a higher energy group of noncovalent bonding bands. Also interesting in this regard are the bonding and antibonding branches within the lower $x^{2}-y^{2}, y z$ orbitals due to residual $\mathrm{Nb}-\mathrm{Nb}$ dimerization, resulting in a $V$-shaped electronic state near $E_{F}$ for the $x^{2}-y^{2}, y z$ orbitals of $\gamma-\mathrm{NbO}_{2}$. Moreover, in Fig. 3 we display the fully optimized electronic structure reconstruction caused when $\beta$ is reduced as compared to the experimental value of $104.29^{\circ}$. This will serve as a reference to highlight the additional effects of pressure on the band structure of 


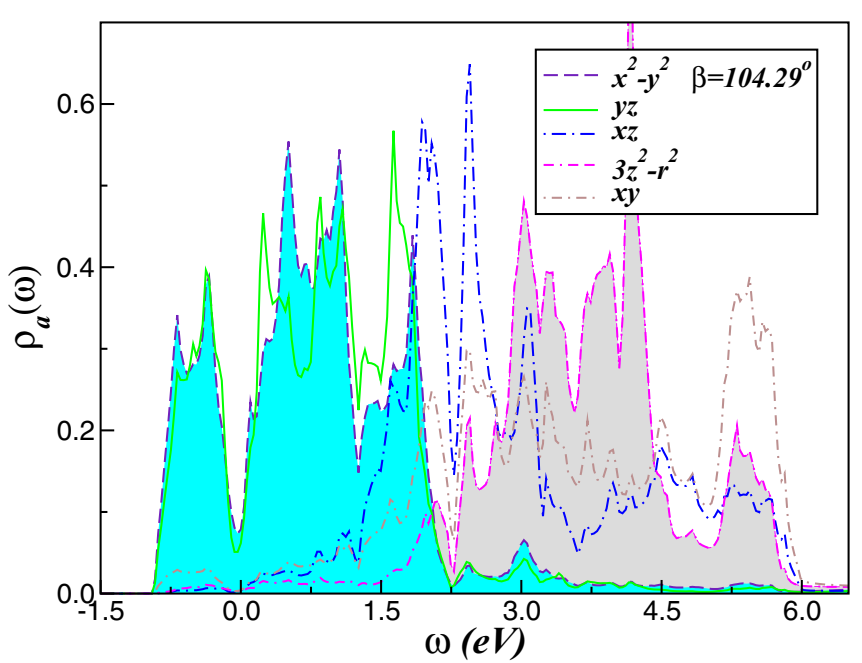

FIG. 2. Orbital-resolved LDA density-of-states (DOS) for the $4 d$ orbitals of (baddeleyite-type) $\gamma-\mathrm{NbO}_{2}$. While all bands span across the Fermi level, $E_{F}=\omega=0$, an important feature to be seen in the $V$-shaped bonding antibonding splitting near $E_{F}$ and the narrow bandwidth of the $x^{2}-y^{2}, y z$ orbitals. This confirms that the electronic states relevant to $\gamma-\mathrm{NbO}_{2}$ have $x^{2}-y^{2}, y z$ orbital character. Noteworthy as well is the dispersive high in energy bands, showing large crystal field splitting effects.

$\gamma-\mathrm{NbO}_{2}$. As seen, in going from $\beta=104.29^{\circ}$ to $99.28^{\circ}$, the $V$-shaped band of the $x^{2}-y^{2}$ orbital is highly reshaped with its tiny band minima being shifted to the conduction band. Also noticeable is the spectral weight transfer between the $x^{2}-y^{2}$ and the $y z$ orbital, implying a gradual change of orbital order pattern [23] with reducing $\beta$.

Based on LDA, the one-electron Hamiltonian part of $\gamma-\mathrm{NbO}_{2}$ is $H_{0}=\sum_{\mathbf{k}, a, \sigma} \epsilon_{a}(\mathbf{k}) c_{\mathbf{k}, a, \sigma}^{\dagger} c_{\mathbf{k}, a, \sigma}+\sum_{i, a, \sigma}\left(\epsilon_{a}^{(0)}-\right.$ u) $n_{i, a, \sigma}$, where $a=\left(x^{2}-y^{2}, y z, x z, 3 z^{2}-r^{2}, x y\right)$ denote the

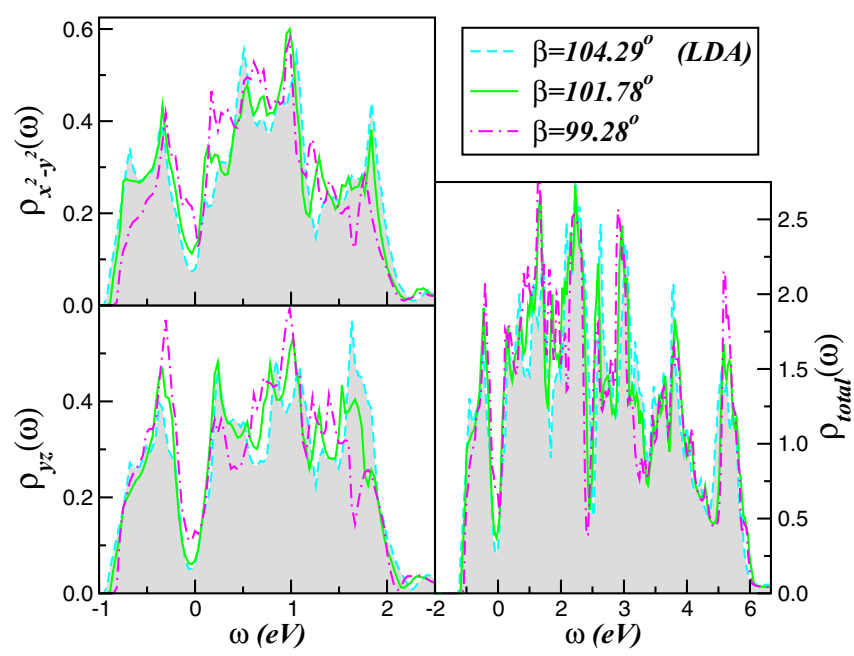

FIG. 3. Effect of reducing the monoclinic angle $\beta$ on the LDA orbital-resolved and total DOS of $\gamma-\mathrm{NbO}_{2}$, showing large changes in the bare band spectra. A particularly interesting feature to be seen is the changes in the $V$-shaped bonding-antibonding band gap and the transfer of spectral weight from the $x^{2}-y^{2}$ to the $y z$ shell of $\gamma-\mathrm{NbO}_{2}$ upon reducing $\beta$ from its experimental value [11]

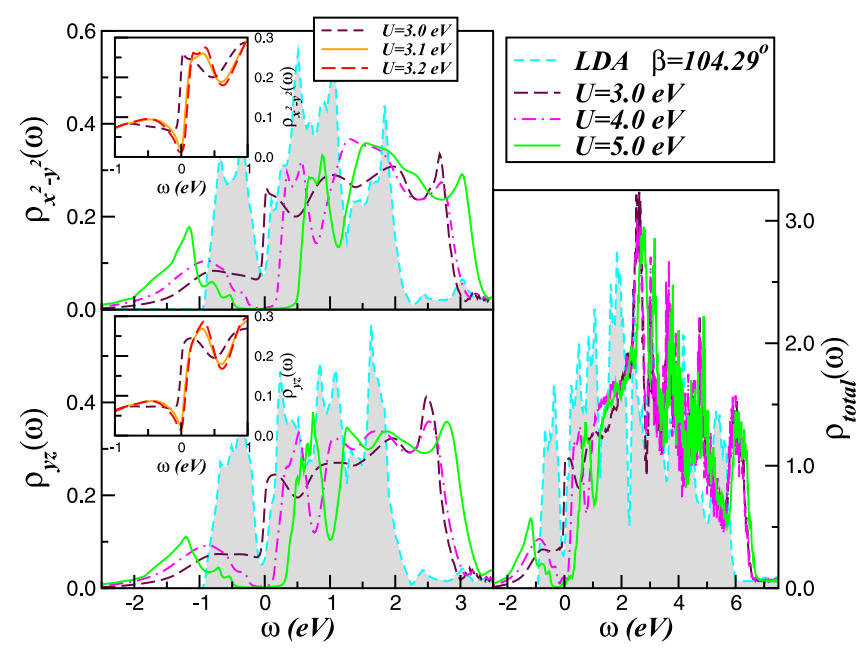

FIG. 4. Comparison between LDA and LDA+DMFT orbitalresolved and total DOS of $\gamma-\mathrm{NbO}_{2}$, showing their evolution with increasing the on-site Coulomb interaction $U$. Apart from the MottHubbard localization, a particularly interesting feature to be seen is the large spectral weight transfer with increasing $U$. Insets show the first-order metal-to-pseudogap phase at the quantum critical $U_{c}=$ $3.1 \mathrm{eV}$. Here, all LDA+DMFT (MO-IPT) spectral functions are computed at zero temperature.

diagonalized $4 d$ orbitals of $\gamma-\mathrm{NbO}_{2}$. In the usual notation, here, $c_{\mathbf{k}, a, \sigma}^{\dagger}\left(c_{\mathbf{k}, a, \sigma}\right)$ are the creation (anihilation) operators for electrons at orbital $a$ and momentum $\mathbf{k}$, with spin $\sigma(=\uparrow$ $, \downarrow)$ and $n_{i, a, \sigma}=c_{i, a, \sigma}^{\dagger} c_{i, a, \sigma}$ is the occupation number operator of an $a$ electron with spin $\sigma$ at site $i . \epsilon_{a}(\mathbf{k})$ is the one-electron band dispersion, which encodes details of the all-electron (LDA) band structure, and the $\epsilon_{a}^{(0)}$ are on-site orbital energies of $\gamma-\mathrm{NbO}_{2}$ whose bare values are read off from LDA spectral functions, and $\mu$ is the chemical potential. These are relevant one-particle inputs for MO LDA+DMFT, which generate a Mott-Hubbard insulating state at $U=$ $4.0 \mathrm{eV}$, as shown below. The correlated many-body Hamiltonian for $\gamma-\mathrm{NbO}_{2}$ reads $H_{\text {int }}=U \sum_{i, a} n_{i, a, \uparrow} n_{i, a, \downarrow}+$ $U^{\prime} \sum_{i, a \neq b} n_{i, a} n_{i, b}-J_{H} \sum_{i, a \neq b} \mathbf{S}_{i, a} \cdot \mathbf{S}_{i, b}$, where $U$ is the onsite Coulomb interaction, $U^{\prime}=U-2 J_{H}$ is the interorbital Coulomb interaction term, and $J_{H}$ is the Hund's coupling. We evaluate the many-particle Green's functions of the MO Hamiltonian $H=H_{0}+H_{\text {int }}$ within LDA+DMFT, [12] using MO iterated perturbation theory (MO-IPT) as the impurity solver [24].

Let us now discuss our LDA+DMFT results obtained within the $d^{1}$ electronic configuration of the $\gamma-\mathrm{NbO}_{2}$ parent compound. In Fig. 4, we display the effect of electronelectron interactions on the active $x^{2}-y^{2}, y z$ orbitals and the total spectral function $\left[\rho_{\text {total }}(\omega)=\sum_{a, \sigma} \rho_{a, \sigma}(\omega)\right]$ [25] of $\gamma-\mathrm{NbO}_{2}$. At $U=3.0 \mathrm{eV}$ (and $J_{H}=1.0 \mathrm{eV}$ ) [26], $\gamma-\mathrm{NbO}_{2}$ is on the metallic side of the correlated phase diagram [1]. On increasing the on-site Coulomb interaction $U$, the MottHubbard insulating state is clearly resolved at $U=4.0 \mathrm{eV}$. Lower Hubbard bands (LHBs) are visible in the two orbitalresolved spectral functions. As seen, the $x^{2}-y^{2}$ orbital shows stronger correlation effects with pronounced LHBs at $U=$ $5.0 \mathrm{eV}$, while the $y z$ orbital has less tendency toward local moment formation (LHB) due to $U^{\prime}$-enhanced orbital 


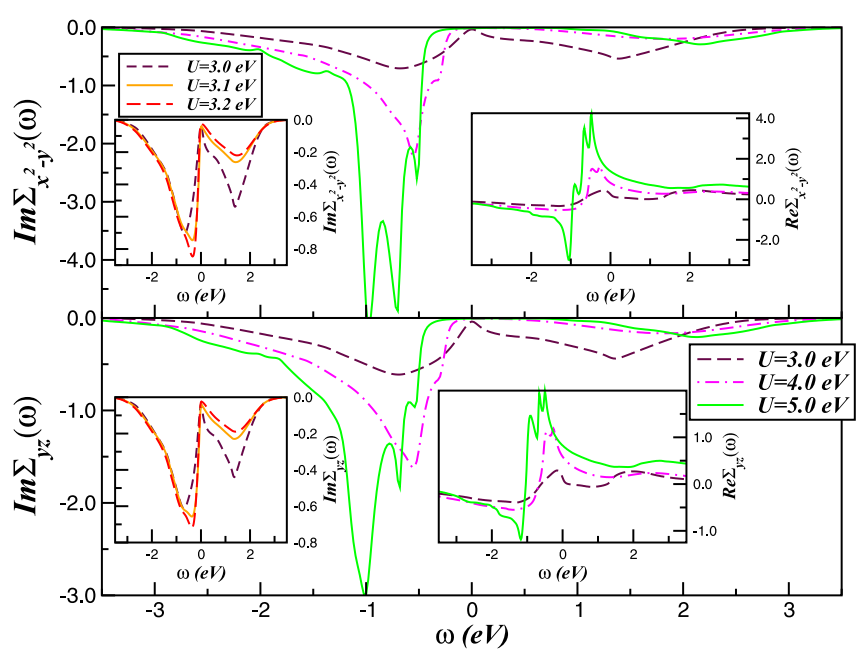

FIG. 5. Orbital-resolved self-energies imaginary (main panels) and real (right insets) parts of $\gamma-\mathrm{NbO}_{2}$, showing their evolution with increasing the on-site Coulomb interaction $U$ for fixed $\beta=104.29^{\circ}$. A particular feature to be seen is the clearly visible particle-hole asymmetry [13]. Left insets show the changes in the self-energy imaginary part near the metal-to-pseudogap phase transition.

polarization effects. Changes in the LDA+DMFT electronic structure are also observed in the total spectral function, showing large-scale changes in spectral weight transfer as compared to LDA with increasing $U$. As seen, in Fig. 4, the LDA+DMFT spectral functions are highly reshaped by sizable MO electron-electron interactions compared to LDA, and future photoemission and x-ray absorption experiments in pressurized $\mathrm{NbO}_{2}$ could verify this aspect.

For a more detailed analysis of dynamical MO correlation effects in $\gamma-\mathrm{NbO}_{2}$, in the insets of Fig. 4 we display the abrupt changes in the spectral functions across the first-order metal-to-pseudogap transition [27]. For the sake of clarity, we recall here that pseudogap state refers to a loss of lowenergy electron excitations due to a depression of electronic states near $E_{F}[28,29]$. As seen in the insets of Fig. 4, MO electronic correlations lead to abrupt modifications of the correlated spectra. Above the critical value of the pseudogap phase $\left(U_{c}=3.1 \mathrm{eV}\right)$, the many-body spectra describe an incoherent, non-Fermi liquid quantum critical system with orbital dependent low-energy pseudogap features. Noteworthy is the suppression of the one-electron dispersion at low energies and the emergence of a well-defined LHB at energies close to $-0.45 \mathrm{eV}$. Also, the shoulder features above $E_{F}$ are clearly reshaped due to an abrupt dynamical transfer of spectral weight. Pseudogap formation within the $4 d$ transition-metal oxide family has been reported for $\mathrm{BaRuO}_{3}$ [30], implying that a similar incoherent metal might be seen in the paramagnetic state of $\gamma-\mathrm{NbO}_{2}$ due to reduced $U / W$ ratio via band broadening with increasing pressure well beyond $8.5 \mathrm{GPa}$ $[11,19]$.

Next, in Fig. 5, we show the $U$ dependence of the selfenergy imaginary (main panels) and real (right insets) parts associated with the active $4 d$ orbitals of $\gamma-\mathrm{NbO}_{2}$. Comparing our results to that reported for the bct phase of $\mathrm{NbO}_{2}$ [13], we notice similar features in both structural phases, i.e., the absence of poles at $E_{F}$ and the strong particle-hole asymmetry in $\Sigma_{a}(\omega)$. This particle-hole asymmetry is manifested by the appearance of a peak close to $-0.5 \mathrm{eV}$ in $\operatorname{Im} \Sigma_{a}(\omega)$, which is strongly enhanced and transferred toward higher binding energies with increasing $U$. Interestingly, such frequency dependence suggests that the Mott instability of $\gamma-\mathrm{NbO}_{2}$ might also be arrested [13] as in the dimerized bct phase. Taken together, our results and those obtained using DFT plus cluster-DMFT calculations [13], a remarkable aspect stands out: $\operatorname{Im} \Sigma_{a}(\omega)$ vanishes in the region of the Mott gap, instead of having a pole, as would occur in a conventional Mott insulator. As pointed out in our earlier studies [31], this aspect is somehow reminiscent of a correlated Mott-Kondo insulator, where the gap arises due to combined effects of strong dynamical correlations and sizable interband and interorbital hybridization inducing (bonding-antibonding) pseudo-band-gaps in the bare electronic structure. Moreover, as can be seen in the right insets, the self-energy real parts have pronounced frequency dependence near $E_{F}$, which indicates that the bare electronic states of the active bands are strongly renormalized by these components as in bet $\mathrm{NbO}_{2}$ [13].

We turn now to the effect of slightly decreasing the monoclinic angle $\beta$ from its experimental value [11] on the correlated electronic structure of $\gamma-\mathrm{NbO}_{2}$ system. Before delving into this problem, it is worth mentioning that under external perturbations like pressure and strain, the hopping elements and the crystal field splittings are renormalized in nontrivial ways. In practice, it is difficult to separate the effects of the hopping from those induced by crystal field splittings, especially if the correlated electronic structure under pressure is not known a priori. With these caveats in mind, here we study the effect of reducing the monoclinic distortion on the correlated many-body states of $\gamma-\mathrm{NbO}_{2}$. To achieve this aim, we use the MO LDA DOS of Fig. 3 to show the effect of lattice distortion on the electronic-structure reconstruction of pressurized $\mathrm{NbO}_{2}$. As seen in Fig. 6 in a correlated electron system, small changes in the bare band structure, including local energy splittings and orbital occupancies, lead to large dynamical spectral weight transfer typically over a scale of a few electron volts. As is clearly visible in Fig. 6, at fixed $U=4.0 \mathrm{eV}$ we observe systematic changes, albeit with different dynamical spectral weight transfer in the orbitalresolved spectral functions with $\beta$. In this pressurized regime, the $x^{2}-y^{2}, y z$ orbitals remain Mott localized, showing small enhancement of the Mott gap size with reducing $\beta$. Particularly interesting in Fig. 6 are the changes in the lower energy spectra at $U=3.0 \mathrm{eV}$ (see insets), showing that a quantum critical behavior (characterized by a deep in the DOS [32]) can be tuned upon small changes in $\beta$ under pressure. Here, we emphasize that the emergence of insulating, pseudogaped, and metallic states under pressure is the key mechanistic step, which allows us to understand the seeds toward unconventional electronic-structure reconstruction of strongly correlated electrons close to Mottness. Hence, it would be interesting to see whether this mechanism might be observable in spectroscopy experiments of pressurized $\mathrm{NbO}_{2}$. Future experimental works to corroborate our prediction are called for.

Additionally, we reveal in Fig. 7 the angle dependence of the self-energy imaginary (main panels) and real (right insets) 


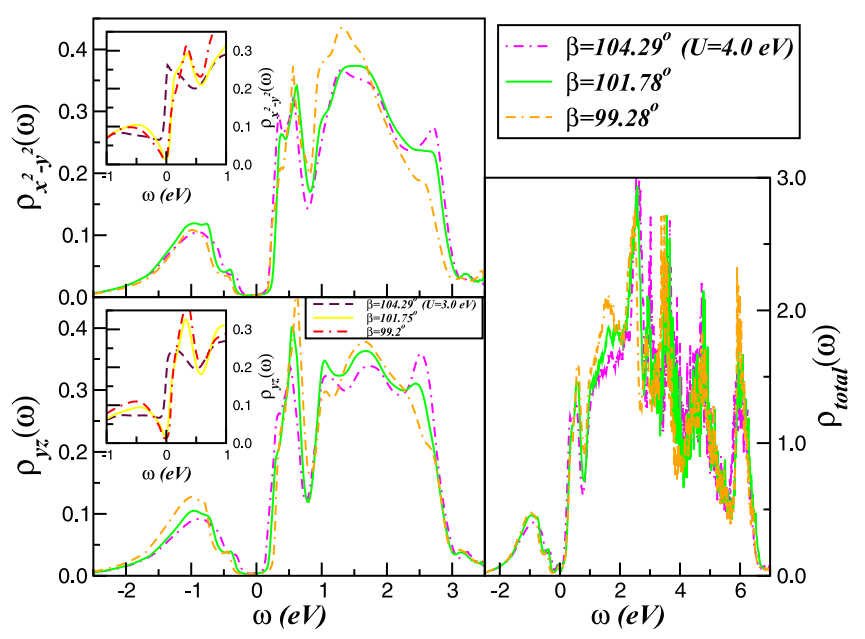

FIG. 6. Effect of the monoclinic angle $\beta$ on the LDA+DMFT orbital-resolved and total DOS of $\gamma-\mathrm{NbO}_{2}$, showing similar strong coupling Mott localization. Insets show the electronic-structure reconstruction close to $U_{c}$ and the importance of $\beta$ in determining the emergence of metallic and pseudogaped electronic states in the high pressure electronic structure of $\mathrm{NbO}_{2}$. Notice the modification of the LDA+DMFT spectral functions toward a pseudogaped quantum critical regime due to large-scale dynamical spectral weight transfer.

parts of $\gamma-\mathrm{NbO}_{2}$, showing similar behavior as in Fig. 5 as well as that reported for the bct structural phase of $\mathrm{NbO}_{2}$ [13]. As seen, at $U=4.0$ the self-energies real parts show nearly similar frequency-dependence. Interesting, however, is the $\beta$ dependence of $\operatorname{Im} \Sigma_{a}(\omega)$, both in the main panels and the left insets. While in the Mott localized regime $\operatorname{Im} \Sigma_{y z}$ shows stronger correlations effects for $\beta=99.28^{\circ}$, the $x^{2}-y^{2}$ orbital sector displays enhanced self-energy corrections for $\beta=$ $101.78^{\circ}$. This suggests that structural distortions play a different important role in the electronic-structure reconstruction of pressurized $\mathrm{NbO}_{2}$. Finally, looking closely at the self-energy

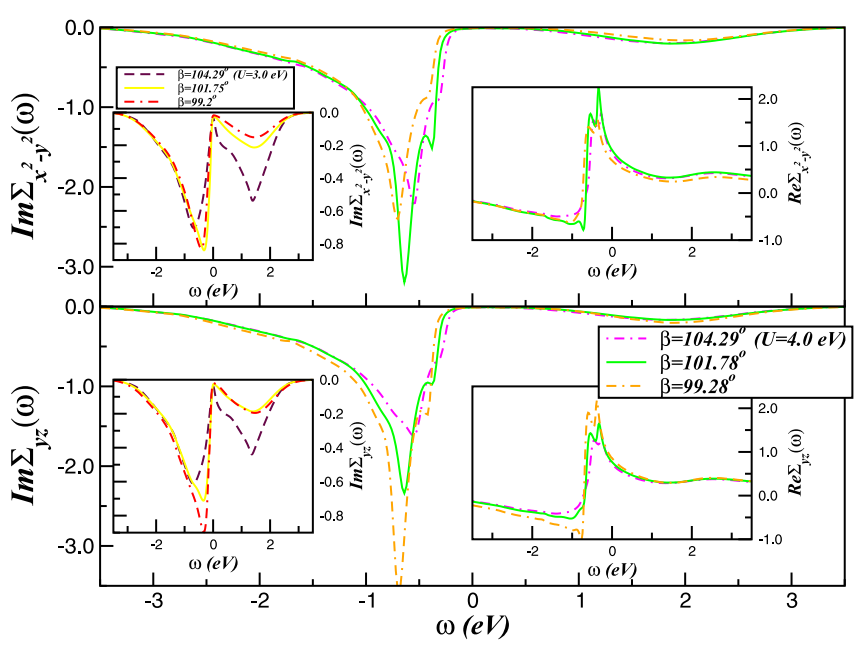

FIG. 7. Effect of the monoclinic angle $\beta$ on the orbital-resolved self-energies imaginary (mail panels) and real (right panels) of $\gamma-\mathrm{NbO}_{2}$ for $U=4.0 \mathrm{eV}$, showing orbital-dependent electron correlation effects upon reducing $\beta$. Left insets display the frequency dependence of the self-energy imaginary parts close to $U_{c}$.

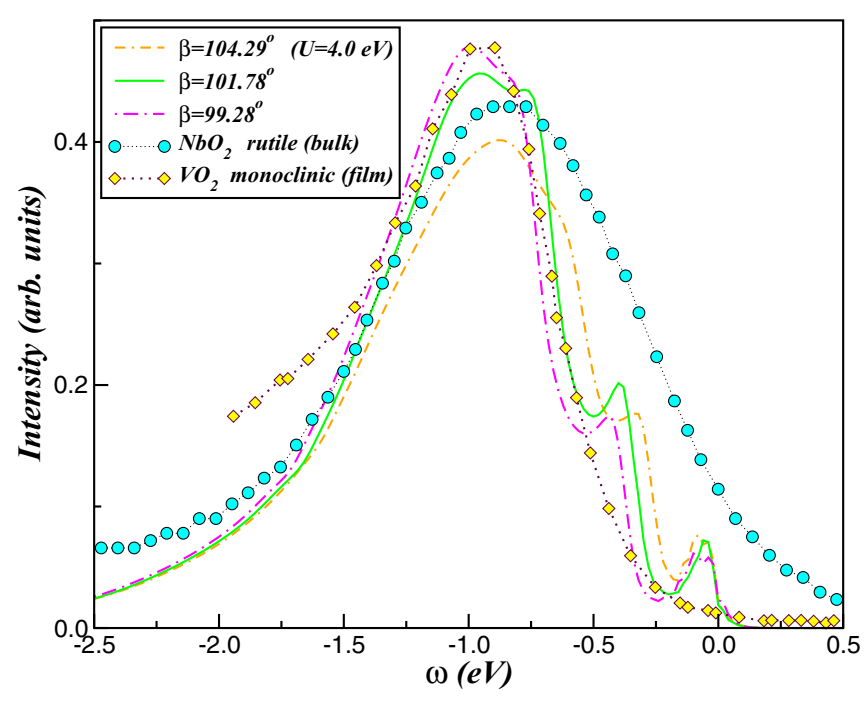

FIG. 8. Theory-experiment comparison between the total LDA+DMFT spectral function of $\gamma-\mathrm{NbO}_{2}$ and hard $\mathrm{x}$-ray photoelectron spectroscopy (HAXPES) spectra taken from Ref. [14], showing qualitative good agreement in spite of different structural phases. Notice the position of the lower Hubbard band, which nicely agrees with experiment. The measured valence band spectra of monoclinic $\mathrm{VO}_{2}$ (10-nm-thin film) [9] is shown for comparison. Notice the lower Hubbard band main peak position, which almost coincides both with theory and experiment in spite of different crystal structures and pressure conditions. This universal behavior suggests that the peak position of the lower Hubbard band is Mott driven and nearly independent of the band structure in early $d^{1}$ transition-metal oxide systems.]

imaginary parts for $U=3.0 \mathrm{eV}$ (see left insets), we notice that particle-hole asymmetry is enhanced upon reducing the monoclinic angle, and this aspect could be verified in future experiments probing the energy dependence of the orbitalresolved scattering rates and quasiparticle weights of $\mathrm{NbO}_{2}$ at high-pressure conditions [33].

To rationalize the overall correlated electronic behavior of $\mathrm{NbO}_{2}$, in Fig. 8 we compare our LDA+DMFT results with extant hard x-ray photoelectron spectroscopy (HAXPES) spectra for the rutile phase [14]. Clearly, good qualitative agreement between theory and experiment is found over the entire range of $-2 \leqslant \omega \leqslant E_{F}$. In particular, the energy position of the LHB as well as the details of the line shape below $0.8 \mathrm{eV}$ binding energy are all in good agreement with experiment in spite of different crystal structures and $\beta$ values. Thus, our theory-experiment comparison represents a qualitatively accurate representation of the valance one-electron spectral function in the paramagnetic phase of $\mathrm{NbO}_{2}$. Lastly, we stress some essential differences between our work and earlier studies. In Refs. [13,14], an $U=6.0 \mathrm{eV}$ was used in their correlated electronic structure calculations, which according to our results in Fig. 4, will sensibly lower the LHB peak in contrast to experiment. Finally, we draw attention to the good qualitative agreement between theory and experiment for the valence band one-electron spectral function obtained using LDA+DMFT. Importantly, we observe a nearly universal behavior in that the peak position of the LHB in the theory and experimental data are independent of crystal- and band- 
structure details, thus achieving universal agreement between experiment and theory for early $d^{1}$ transition-metal oxide systems

\section{CONCLUSION}

In summary, we performed LDA+DMFT calculations to investigate the role of dynamic electronic correlations on the electronic-structure reconstruction of pressurized (baddeleyite-type) $\mathrm{NbO}_{2}$, focusing on the mechanism hidden in the Mott metal-insulator transition and the comparison between our results and extant experimental data for the rutile phase at ambient pressure. Our theory-experiment comparison shows that the energy position of the LHB is one of the universal electron correlation effects on the valence band spectrum of early transition-metal oxides. In particular, for pressurized $\mathrm{NbO}_{2}$ we find that the correlation effects are more pronounced upon reduction of the monoclinic angle, although the calculated spectral functions show that electronic dynamical correlations induce nearly similar LHBs. Moreover, we find that dynamical correlations play an important role in the gap formation of baddeleyite-type $\mathrm{NbO}_{2}$, showing a first-order metal-to-pseudogap transition [27] at a quantum critical Coulomb interaction value, which can be tuned by changing the monoclinic angle. The interplay between pseudogap-induced fermionic incoherence in the paramagnetic normal state and the changes in the monoclinic angle under pressure suggests a promising but also practical route to access quantum criticality in pressurized dimer quantum systems [34].

\section{ACKNOWLEDGMENTS}

L.C.'s work is supported by CNPq (Grant No. 304035/2017-3). Acknowledgment (L.C.) is also made to CAPES. S.L. thanks ARCCA Cardiff for computational resources. Via S.L.'s membership of the UK's HPC Materials Chemistry Consortium, which is funded by EPSRC (No. EP/L000202), this work made use of the facilities of ARCHER, the UK's National High-Performance Computing Service, which is funded by the Office of Science and Technology through EPSRC's High End Computing Programme.
[1] M. Imada, A. Fujimori, and Y. Tokura, Rev. Mod. Phys. 70, 1039 (1998).

[2] F. J. Morin, Phys. Rev. Lett. 3, 34 (1959); J. Park, L. H. Tjeng, A. Tanaka, J. W. Allen, C. T. Chen, P. Metcalf, J. M. Honig, F. M. F. de Groot, and G. A. Sawatzky, Phys. Rev. B 61, 11506 (2000); R. Basu, V. Srihari, M. Sardar, S. Kumar Srivastava, S. Bera, and S. Dhara, Sci. Rep. 10, 1977 (2020).

[3] Z. Shao, X. Cao, H. Luo, and P. Jin, NPG Asia Mater. 10, 581 (2018).

[4] A. Zylbersztejn and N. F. Mott, Phys. Rev. B 11, 4383 (1975).

[5] J. B. Goodenough, J. Solid State Chem. 3, 490 (1971).

[6] S. Biermann, A. Poteryaev, A. I. Lichtenstein, and A. Georges, Phys. Rev. Lett. 94, 026404 (2005); C. Weber, D. D. O’Regan, N. D. M. Hine, M. C. Payne, G. Kotliar, and P. B. Littlewood, ibid. 108, 256402 (2012).

[7] M. S. Laad, L. Craco, and E. Müller-Hartmann, Europhys. Lett. 69, 984 (2005); Phys. Rev. B 73, 195120 (2006).

[8] W. H. Brito, M. G. O. Aguiar, K. Haule, and G. Kotliar, Phys. Rev. Lett. 117, 056402 (2016).

[9] D. Shiga, B. E. Yang, N. Hasegawa, T. Kanda, R. Tokunaga, K. Yoshimatsu, R. Yukawa, M. Kitamura, K. Horiba, and H. Kumigashira, arXiv:2005.00304.

[10] J. Chakhalian, J. W. Freeland, H.-U. Habermeier, G. Cristiani, G. Khaliullin, M. van Veenendaal, and B. Keimer, Science 318, 1114 (2007).

[11] J. Haines, J. M. Léger, A. S. Pereira, D. Häusermann, and M. Hanfland, Phys. Rev. B 59, 13650 (1999).

[12] G. Kotliar, S. Y. Savrasov, K. Haule, V. S. Oudovenko, O. Parcollet, and C. A. Marianetti, Rev. Mod. Phys. 78, 865 (2006).

[13] W. H. Brito, M. C. O. Aguiar, K. Haule, and G. Kotliar, Phys. Rev. B 96, 195102 (2017).

[14] W.-C. Lee, M. J. Wahila, S. Mukherjee, C. N. Singh, T. Eustance, A. Regoutz, H. Paik, J. E. Boschker, F. Rodolakis,
T.-L. Lee, D. G. Schlom, and L. F. J. Piper, J. Appl. Phys. 125, 082539 (2019).

[15] V. Eyert, Europhys. Lett. 58, 851 (2002).

[16] See, F. Grandi, A. Amaricci, and M. Fabrizio, Phys. Rev. Res. 2, 013298 (2020) and references therein.

[17] R. F. Janninck and D. H. Whitmore, J. Phys. Chem. Solids 27, 1183 (1966); T. Sakata, K. Sakata, and I. Nishida, Phys. Stat. Sol. 20, K155 (1967).

[18] A. K. Cheetham and C. N. R. Rao, Acta Cryst. B 32, 1579 (1976); R. Pynn, J. D. Axe, and R. Thomas, Phys. Rev. B 13, 2965 (1976).

[19] T. Okamoto and S. Minomura, J. Phys. Soc. Jpn. 50, 3661 (1981).

[20] A. O'Hara and A. A. Demkov, Phys. Rev. B 91, 094305 (2015).

[21] B. Bondars, G. Heidemane, J. Grabis, K. Laschke, H. Boysen, J. Schneider, and F. Frey, J. Mater. Sci. 30, 1621 (1995).

[22] O. K. Andersen, Phys. Rev. B 12, 3060 (1975).

[23] S. Leoni, L. Craco, A. Ormeci, and H. Rosner, Solid State Sci. 8, 1138 (2006).

[24] L. Craco, Phys. Rev. B 77, 125122 (2008).

[25] $\rho_{a, \sigma}(\omega) \equiv-\frac{1}{\pi} \sum_{\mathbf{k}} G_{a, \sigma}(\mathbf{k}, \omega), \quad$ with $\quad G_{a, \sigma}(\mathbf{k}, \omega)=$ $\frac{1}{\omega+\mu-\Sigma_{a, \sigma}-\epsilon_{a}(\mathbf{k})}$ being the retarded one-particle Green's function of orbital $a$.

[26] We use the same $J_{H}$ value as in Refs. [13, 14].

[27] J. P. L. Faye and D. Sénéchal, Phys. Rev. B 96, 195114 (2017).

[28] M. R. Norman, D. Pines, and C. Kallin, Adv. Phys. 54, 715 (2005).

[29] G. Sordi, P. Sémon, K. Haule, and A.-M. S. Tremblay, Phys. Rev. Lett. 108, 216401 (2012).

[30] Y. S. Lee, J. S. Lee, K. W. Kim, T. W. Noh, J. Yu, E. J. Choi, G. Cao, and J. E. Crow, Europhys. Lett. 55, 280 (2001).

[31] L. Craco, M. S. Laad, and S. Leoni, J. Phys.: Condens. Matter. 26, 145602 (2014); B. Freelon, Y. Hao Liu, J.-L. Chen, L. 
Craco, M. S. Laad, S. Leoni, J. Chen, L. Tao, H. Wang, R. Flauca, Z. Yamani, M. Fang, C. Chang, J.-H. Guo, and Z. Hussain, Phys. Rev. B 92, 155139 (2015).

[32] A. Abanov, Y.-M. Wu, Y. Wang, and A. V. Chubukov, Phys. Rev. B 99, 180506(R) (2019).
[33] See, L. Craco and S. Leoni, Phys. Rev. B 101, 245133 (2020) and references therein.

[34] P. Merchant, B. Normand, K. W. Krämer, M. Boehm, D. F. McMorrow, and Ch. Rüegg, Nat. Phys. 10, 373 (2014). 